\title{
Requerimientos informacionales para la toma de decisiones estratégicas en organizaciones de información
}

\author{
Information requirements for strategic decision making \\ in information organizations
}

Yunier RODRÍGUEZ-CRUZ1

María PINTO

\section{Resumen}

Se examina el carácter informacional del proceso de toma de decisiones estratégicas con el propósito de identificar los requerimientos informacionales que condicionan el uso de información que se realiza en los niveles estratégicos en instituciones de información. El acercamiento teórico al tema permite comprender las particularidades en relación a la información como recurso estratégico y determinar aquellos factores y elementos que desde las Ciencias de la Información han contribuido a la teoría de las decisiones organizacionales. El estudio se realizó con directivos de once instituciones cubanas. Se profundiza, a partir del método fenomenográfico, en la opinión de los entrevistados acerca de los recursos, procesos, sistemas, factores y estados emocionales vinculados a la información y su uso en la toma de decisiones. Como resultado se identifica un conjunto de requerimientos informacionales, entre los que destacan los sistemas de información, procesos informacionales y gerenciales, así como un conjunto de recursos informacionales y factores socio-cognitivos que posibilitan fomentar y generar una infraestructura informacional y potenciar la cultura informacional necesaria para el desarrollo de procesos de decisión eficaces a nivel estratégico.

Palabras clave: Fenomenografía. Requerimientos informacionales. Toma de decisiones estratégicas. Uso de información.

\begin{abstract}
The informational character of the strategic decision-making process is examined with the purpose of identifying the informational requirements that influence the use of information in the strategic levels of information organizations. The theoretical approach to these topics allows one to understand the information particularities as a strategic resource and to determine the elements of Information Sciences that have contributed to the theory of organizational decisions. The study was conducted with executives from eleven Cuban institutions. The phenomenographic method was used to explore the opinion of the interviewees about the resources, processes, systems, factors and emotional states linked to information and its use in the decision-making process. As a result, a set of informational requirements was identified, such as information systems, informational and management processes, as well as a set of informational resources and socio-cognitive factors that make it possible to foster and generate an information infrastructure and enhance the information culture required for the development of strategic decision-making processes.
\end{abstract}

Keyword: Phenomenography. Information requirement. Srategic decision making. Information use.

\footnotetext{
1 Universidad de La Habana, Facultad de Comunicación, Departamento Ciencias de la Información. Edificio Bohemia s/n., e/ Ermita y San Pedro, Plaza de la Revolución, La Habana, Cuba. Correspondencia a nombre de/Correspondence to: Y. RODRÍGUEZ-CRUZ. E-mail: <yunier@fcom.uh.cu>.

2 Universidad de Granada, Facultad de Biblioteconomía y Documentación, Departamento de Información y Comunicación. Paseo de Cartuja, s/n., 18071, Granada, España.

Recibido el día 18/11/2015, re-presentado el 2/9/2016 y aceptado para su publicación el 14/12/2016.
} 


\section{Introdución}

La toma de decisiones estratégicas constituye un tema de interés en tanto "las decisiones estratégicas son la preocupación central para las organizaciones modernas" (MITCHELL et al., 2011, p.697), ya que a través de éstas "es posible la creación de valor estratégico" (RODRÍGUEZ; PEDRAJA, 2009, p.34) en ambientes organizacionales cada vez más dinámicos y cambiantes.

Una adecuada comprensión y mejora de este proceso conlleva, por tanto, profundizar en sus componentes particulares o intrínsecos: 'situaciónproblema', 'individuo', 'información'y 'contexto', aspectos que han sido abordados entre otros por Angeloni (2003), Caixeta y Rodrigues (2008), Howard y Ortiz (1971), Rodríguez-Cruz y Pinto (2010).

No obstante, si bien la interrelación e integración de cada componente es la que incide de forma favorable en los procesos de decisión, autores como Allen (2011), Bettis (2012), Citroen (2011), Fiol (2001) y Valentim (2006) entre otros, han validado la importancia de la información en los procesos de decisión, enfatizando la noción de que sin un adecuado uso y tratamiento de este recurso, no se podrían tomar decisiones con la calidad requerida y que posibiliten la solución de problemas, aprovechamiento de oportunidades y prevención de riesgos.

En consecuencia, y dado el carácter informacional de los procesos de decisión, el siguiente estudio tiene como objetivo identificar los requerimientos informacionales que contribuirían, de manera significativa, a una eficiente y eficaz toma de decisiones. El cumplimiento del mismo se sustenta en la aplicación del método fenomenográfico y el análisis de contenido para la identificación de dichos requerimientos. A partir de este método cualitativo de investigación se puede profundizar sobre aquellos elementos o componentes que pueden incidir favorablemente en un mejor uso de información en los niveles de decisión estratégicos.

\section{La toma de decisiones estratégicas, sus particularidades y carácter informacional}

La toma de decisiones organizacionales se consolida como teoría de la administración en la década del '50 del pasado siglo XX por autores entre los que se encuentra Herbert A. Simon [1916-2001], considerado uno de sus fundadores. Ya a inicios de los años '60 son varios los autores y las áreas de conocimiento que han intentado definir y conceptualizar este proceso. Entre los primeros se encuentran autores como: SIMON (1955), MINTZBERG et al. (1976). Entre las ciencias particulares se destacan la Antropología, Sociología, Psicología, Economía y la Gestión.

La toma de decisiones organizacionales "constituye un proceso de información que se desarrolla por individuos o grupos para solucionar problemas y aprovechar oportunidades organizacionales. En éste se analizan, mediante la consecución de determinadas etapas, un conjunto de alternativas de solución/ decisión para identificar la mejor de ellas, de forma que el decisor pueda desplazarse de una posición actual, en la que se encuentra, a la posición en la que desea estar (ideal), en consecuencia con las metas, objetivos y razón de ser de la organización" (RODRÍGUEZ-CRUZ; PINTO, 2010, p.67). No obstante, entre los términos que priman en los conceptos de toma de decisiones organizacionales se encuentran indistintamente: proceso organizacional, información, individuo(s), etapas o procesos, decisión, acción, alternativas de decisión, solución de problemas, aprovechamiento de oportunidades y valor estratégico.

En relación a este último, el mismo ha sido uno de los elementos que han influido en el creciente interés por las decisiones estratégicas, las que "constituyen elecciones cuyo impacto es de largo alcance [...] estas elecciones involucran el empleo de recursos significativos de las instituciones, e implican la participación de varios niveles y funciones dentro de las organizaciones" (RODRíGUEZ; PEDRAJA, 2009, p.34).

La toma de decisiones estratégicas constituye, por tanto, un proceso informacional en el que el(los) directivo(s) del máximo nivel de dirección de una organización eligen y conciben, mediante una serie de etapas, la mejor alternativa de decisión y su curso de acción, para solucionar un problema y aprovechar una oportunidad que pueda afectar o incidir en la estrategia organizacional y por ende en la proyección estratégica de la organización en su ambiente de negocios. 
En la consideración de autores como Jansen et al. (2011), McKenzie et al. (2011), Rodríguez-Cruz y Pinto (2010), Wilson et al. (2010), los elementos distintivos de la toma de decisiones estratégicas son:

- Constituye un proceso que se orienta a la organización y su contexto, examinando sus relaciones y dinámicas de interacción.

- El contexto y su adecuada percepción determinan el proceso.

- Genera elevados grados de riesgo e incertidumbre.

- En su desarrollo se asumen grandes responsabilidades y compromisos para con la organización.

- Implican más riesgos, y por tanto más consecuencias.

- Intervienen actores y factores internos y externos a la organización.

- La información que se usa proviene de fuentes externas e internas que por su carácter estratégico exigen su validación para garantizar criterios de calidad y confiabilidad.

En relación al vínculo contexto-organización, varios autores han identificado varias características particulares que inciden en la toma de decisiones estratégicas: 'dominio de emergencias' y 'capacidades dinámicas organizacionales' (MCKENZIE et al., 2011), 'trabajo en equipo o grupos de decisión' (CHOU et al., 2012), (JANSEN et al., 2011), 'fuentes de información estratégicas y no estratégicas' (CITROEN, 2011) y la denominada 'sobrecarga de información' (BETTIS, 2012).

El 'dominio de emergencias' y 'los grupos de decisión' están interrelacionados pues el primero constituye una dinámica de decisión que implica prueba, pensamiento y respuesta ante determinada emergencia o escenario. En este dominio los jugadores claves son los líderes y gerentes de grupos conformados por expertos. Estos grupos y su interacción son los que desarrollan la toma de decisiones y son imprescindibles ya que "la toma de decisiones estratégica requiere cooperación entre miembros de un equipo, pues necesitan desarrollar cognición en equipo para trasladar sus roles y colaboración respectivos sobre las tareas de los procesos de toma de decisión estratégica" (CHOU et al., 2012, p.383).

En relación a las 'capacidades dinámicas organizacionales', este concepto se refiere a los recursos y estrategias para el adecuado desarrollo de la toma de decisiones que le garantice a las organizaciones permanecer, destacarse, prosperar y adaptarse a ambientes cada vez más cambiantes.

Las fuentes de información estratégicas y no estratégicas, así como la información del ambiente externo, son determinantes en los niveles estratégicos de decisión. Citroen (2011) identifica entre las fuentes estratégicas a los suministradores, socios, clientes o usuarios, así como los competidores, que en conjunto conforman la cadena de suministro de las organizaciones. La información proveniente de las mismas se obtiene a través del monitoreo o vigilancia estratégica hacia el ambiente externo de la organización.

Por su parte, los efectos por la carga excesiva de información en la toma de decisiones ha sido un fenómeno abordado por Bettis (2012), quien la define como "sobrecarga de información" visualizada como un constructo multidimensional, constituido por tres componentes: equívoco, cantidad y variedad. Éstos se asocian a las múltiples interpretaciones que se pueden realizar de la información, su cantidad y acceso y la variedad de recursos o fuentes informacionales. Este concepto es de suma importancia pues disponer de mucha y diversa información puede atentar, en ocasiones, contra la capacidad de procesamiento y análisis de información que tiene lugar en el proceso de decisión a nivel estratégico.

A estas particularidades de la toma de decisiones estratégicas se suma su carácter informacional. En este sentido es necesario destacar que si bien la importancia de este recurso ha sido abordada por múltiples autores, los "aspectos sobre el rol de la información en los procesos de toma de decisiones reciben poca atención en investigaciones gerenciales" (CITROEN, 2011, p.493).

No obstante, el carácter informacional de los procesos de decisión está condicionado por la propia dinámica de interrelación de los componentes intrínsecos para la toma de decisiones. En este 
sentido, una adecuada representación de la situaciónproblema depende de una buena percepción de la información que ésta genera, sus causas y efectos. Por otra parte, los individuos toman decisiones al desarrollar procesos informacionales que requieren determinadas capacidades y competencias. No por último menos importante, todas las decisiones organizacionales se tienen que contextualizar, lo que se logra cuando se tiene dominio de la información sobre el funcionamiento del ambiente interno y externo de la organización.

Se puede precisar entonces, una vez examinados determinados enfoques y criterios de autores como Bettis (2012), Citroen (2011), Laudon y Laudon (2012), Simon (1955), que el carácter informacional de la toma de decisiones organizacionales está dado por:

- Calidad y Cantidad de la información en los procesos de decisión.

- Capacidad a partir del uso de información para reducir incertidumbre.

- Reconocimiento de la información como recurso estratégico de decisión y no como un insumo.

- Necesidad de competencias informacionales en los directivos para desarrollar procesos de decisión.

- Dependencia de la adecuada ejecución de procesos informacionales: búsqueda y selección, procesamiento, almacenamiento y análisis fundamentalmente.

- Incidencia en la calidad de los procesos de decisión de recursos y sistemas informacionales.

- Necesidad de sistemas de información de apoyo a la toma de decisiones en los tres niveles de decisión organizacional.

- La racionalidad limitada en los procesos de decisión está determinada por el procesamiento y uso de información.

- Los costos de la toma de decisiones dependen en gran medida de la información y los procesos informacionales.

- El tiempo en la toma de decisiones está condicionado por la información y su uso.

La importancia e influencia de la información como recurso estratégico permiten visualizar la toma de decisiones como un proceso estratégico e informacional que requiere contar con condiciones objetivas y subjetivas que optimicen el proceso de decisión. De igual forma, autores como Akgun et al. (2007), Martins y Miranda (2011), Ponjuán et al. (2014), Wright (2013) relacionan este proceso estratégico a procesos gerenciales como la Gestión Documental, Gestión de Información, del Conocimiento, así como la Inteligencia Organizacional, enfatizando el carácter informacional de este proceso.

\section{Procedimentos metodológicos}

El estudio se realiza mediante la aplicación del método de investigación fenomenográfico. La Fenomenografía constituye un método de investigación cualitativa que se desarrolla con individuos, con el objetivo de conocer su percepción sobre determinado fenómeno, por lo que se desarrolla a partir de criterios y valoraciones cualitativas emitidas por éstos. Según Marton (DIEHM; LUPTON, 2012, p.218), este método constituye "un acercamiento a la investigación cualitativa e interpretativa para mapear cualitativamente diferentes formas en que la experiencia humana, conceptualiza, percibe y entiende varios aspectos de un fenómeno en el mundo que los rodea". Por lo tanto, el presente se trata de un estudio cualitativo enfocado "a comprender y profundizar los fenómenos, explorándolos desde la perspectiva de los participantes en un ambiente natural y en relación con el contexto" (HERNÁNDEZ et al., 2010, p.364).

La aplicación del método fenomenográfico permitió identificar los elementos y componentes imprescindibles para un buen uso de información en la toma de decisiones estratégicas. Este resultado se obtuvo a partir de una serie de fases o etapas fundamentales en la investigación. La primera etapa se orientó a determinar el objeto de estudio: la información en la toma de decisiones estratégicas. En una segunda fase se identificaron los individuos involucrados en la investigación, los que aportaron los criterios y opiniones a partir de los que se obtuvieron los resultados fundamentales. En este caso particular se trata de once directivos de nivel estratégico de organizaciones de información. En una tercera etapa se identificaron las categorías de análisis del objeto 
de estudio, que constituyen la base para obtener y procesar los diversos criterios y valoraciones de los directivos entrevistados. En una cuarta fase se procedió a procesar la información obtenida de los entrevistados para posteriormente determinar los principales resultados de investigación y su visualización a través de un mapa conceptual discursivo.

Una vez identificadas las principales características del objeto de estudio de la presente investigación: la información y su uso en la toma de decisiones estratégicas, se seleccionó la muestra de directivos a participar en el estudio. La muestra utilizada es de tipo intencional y consta de once directivos de nivel estratégico de organizaciones de información cubanas de amplio reconocimiento por su impacto en el desarrollo socioeconómico del país (Cuadro 1).

Una vez identificada la muestra de directivos se procedió a determinar las trece categorías analizadas en el estudio. Éstas se identificaron a partir de la revisión de la literatura especializada en el tema y se corresponden con los criterios que intervienen en el uso de información en los procesos de decisión estratégicos. A continuación se presentan las categorías, los autores influyentes y criterios en que se sustentan:
- Particularidades de la toma de decisiones estratégicas: La toma de decisiones estratégicas presenta características particulares en las que incide la información como recurso estratégico.

- Rol de la información: La información incide y es indispensable en la eficacia y eficiencia del proceso de decisión.

- Información crítica o relevante: A nivel estratégico existen diversos tipos de información que aseguran, mediante su uso, una adecuada toma de decisiones.

- Problemáticas asociadas a la información: Existe un conjunto de problemas asociados al uso y manejo de la información que influye en la toma de decisiones estratégicas.

- Factores organizacionales que intervienen en el uso de información: Las organizaciones y sus dinámicas reales de funcionamiento pueden incidir en el uso de información para tomar decisiones.

- Procesos informacionales: La toma de decisiones tiene lugar a partir de los procesos informacionales que se desarrollan en la misma.

- Procesos gerenciales de apoyo: Existen procesos gerenciales que favorecen el uso de la información en la toma de decisiones.

Cuadro 1. Directivos y organizaciones de información.

\begin{tabular}{|c|c|c|c|}
\hline Directivos & Años de Experiencia & Organizaciones de Información & Sector al que pertenece \\
\hline Subdirectora & 5 años & Biblioteca Nacional de Cuba "José Marti" & Sistema Nacional de Bibliotecas \\
\hline Directora & 3 años & $\begin{array}{l}\text { Biblioteca Central de la Universidad de La } \\
\text { Habana "Rubén Martínez Villena" }\end{array}$ & $\begin{array}{l}\text { Universidad de La Habana (Ministerio } \\
\text { de Educación Superior) }\end{array}$ \\
\hline Directora & 4 años & $\begin{array}{l}\text { Biblioteca Pública de La Habana "Ruben } \\
\text { Martínez Villena" }\end{array}$ & $\begin{array}{l}\text { Sistema Nacional de Bibliotecas } \\
\text { Públicas }\end{array}$ \\
\hline Directora & 11 años & Centro de Información Bancaria y Económica & Banco Central de Cuba \\
\hline Subdirectora & Más de 20 años & Centro de Información de la Salud (INFOMED) & Ministerio de Salud Pública \\
\hline Directora & 1 año & Biblioteca Médica Nacional & Ministerio de Salud Pública \\
\hline Directora & 8 años & $\begin{array}{l}\text { Sistema Archivístico Institucional de Salud } \\
\text { Pública }\end{array}$ & Ministerio de Salud Pública \\
\hline Directora (2007-2012) & 5 años & $\begin{array}{l}\text { Instituto de Información Científica y } \\
\text { Tecnológica }\end{array}$ & $\begin{array}{l}\text { Ministerio de Ciencia, Tecnología y } \\
\text { Medio Ambiente }\end{array}$ \\
\hline Directora & 10 años & Consultoría de Información "Delfos" & $\begin{array}{l}\text { Ministerio de la Informática y las } \\
\text { Comunicaciones }\end{array}$ \\
\hline Director & $\begin{array}{l}6 \text { años (Subdirector y } \\
\text { Director) }\end{array}$ & Consultoría de Información "BIOMUNDI" & $\begin{array}{l}\text { Ministerio de Ciencia, Tecnología y } \\
\text { Medio Ambiente }\end{array}$ \\
\hline Directora & 7 años & Centro de Información de la Prensa & Sector de la Prensa \\
\hline
\end{tabular}

Fuente: Elaboración propia (2014). 
- Estados emocionales: Los estados emocionales inciden en el uso y manejo de información de la toma de decisiones.

- Sistemas de Información: Los sistemas de información de apoyo a la toma de decisiones contribuyen a un mejor desarrollo del proceso.

- Técnicas y herramientas: Las técnicas y herramientas para la toma de decisiones estratégicas dependen de un acertado uso y manejo de información.

- Competencias informacionales: La toma de decisiones requiere que los directivos posean competencias informacionales.

- Profesional de la información - Roles informacionales: Los roles informacionales que asumen los individuos en la toma de decisiones los representan como especialistas de información.

A partir de estas categorías de análisis se elaboró una entrevista semiestructurada para la recolección de información, y la obtención de las valoraciones y opiniones de los directivos de las organizaciones de información involucrados en el estudio. El proceso de entrevistas se realizó en sus contextos organizacionales y se caracterizó por ofrecer comodidad a los entrevistados a la hora de exponer ideas y fundamentarlas. Las entrevistas se desarrollaron en el período comprendido entre Mayo del 2013 a Febrero del 2014. Una vez recopilada la información se utilizó el análisis de contenido para su procesamiento. En este propósito se identificaron las opiniones de los entrevistados en cada sesión de trabajo y se codificaron en "afirmaciones", "argumentos" $y$ "ejemplos". Posteriormente se organizaron por categorías de análisis y se procedió a jerarquizar la información otorgándole mayor peso a las afirmaciones y opiniones de cada entrevistado, de forma que los argumentos y ejemplos permitieran, en un segundo nivel de análisis, fundamentar los criterios expuestos. Por último se identificaron descriptores conceptuales de cada criterio ofrecido y se determinó su nivel de aparición y posicionamiento, así como su nivel de relación, para poder conformar un mapa conceptual discursivo que refleje premisas y requerimientos lógicos para proyectar un buen uso de información en la toma de decisiones estratégicas (Figure 1).

\section{Fenomenografía (método de investigacón cualiativo)}

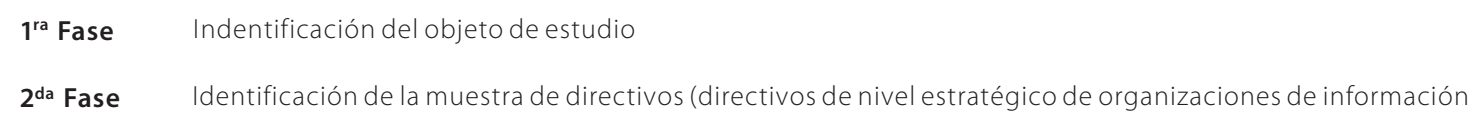

Figure 1. Fases o etapas de la metodología empleada.

Fuente: Elaboración propia (2014). 


\section{Resultados}

Los principales resultados de la investigación se obtienen a partir de los criterios y percepciones de los directivos entrevistados, los que permiten construir un mapa conceptual discursivo de los elementos implicados. De un total de ciento cuarenta criterios que sustentan el estudio, entre los que se encuentran afirmaciones, argumentos y ejemplos, se presentan aquellas opiniones que permiten arribar a resultados a partir de su convergencia, si bien es de destacar que se tienen en cuenta criterios que pueden reflejar otras visiones y que por su importancia se tienen en cuenta en el análisis realizado, aspecto válido que caracteriza a las investigaciones que se apoyan en la Fenomenografía.

A continuación se presentan los resultados obtenidos en/por cada categoría examinada. Se presentan, resultado del análisis de contenido efectuado, las consideraciones y requerimientos informacionales a los que se arriba, y a continuación se explicitan algunos criterios textuales que por su importancia fundamentan los resultados obtenidos.

\section{Particularidades de la toma de decisiones estratégicas}

La toma de decisiones estratégicas, según refieren los entrevistados, debe destacarse por ser un proceso que requiere una previa planificación (100\%), en el que la información se reconoce como un recurso estratégico (100\%), se debe desarrollar de forma grupal (55\%), y que se oriente indistintamente a problemas, oportunidades y riesgos organizacionales (90\%) teniendo en cuenta los objetivos estratégicos de la organización (100\%). A continuación se presentan algunos de los criterios que permitieron identificar estas características:

La toma de decisiones estratégicas debe ser un proceso que se planifique, debe ser formal y estar condicionada por procedimientos claros (Entrevistado 3); [...] debe tener un carácter colectivo o grupal porque permite que los que toman decisiones compartan información y generen nuevo conocimiento. También permite que los mismos sientan una responsabilidad compartida (Entrevistado 6); [...] La información, las fuentes que se utilizan y su validación es fundamental e imprescindible pues la información es clave para tomar decisiones (Entrevistado 11); [...] Identificar un problema de forma clara permite tener ya una visión estratégica (Entrevistado 5); [... L La toma de decisiones debe estar condicionada por los objetivos estratégicos de la organización y va a depender de las características del contexto en que se desarrolla (Entrevistado 2).

\section{Rol de la información}

La información se reconoce por la totalidad de los directivos (100\%) como un recurso estratégico indispensable para todo proceso de decisión. La importancia de la misma radica en que permite comprender la situación-problema, sus causas y efectos, así como contextualizar lo que está ocurriendo una vez que se gestiona información organizacional interna y externa. Otro elemento a destacar es la validación de la información (100\%), pues garantiza se pueda disponer de información confiable, veraz, auténtica y verificable. Los directivos entrevistados ofrecen varios criterios que fundamentan los resultados obtenidos, entre los mismos se encuentran:

Sin información no se puede tomar decisiones pues es el insumo que permite tomar una decisión (Entrevistado 8); [...] Una organización para poder tomar decisiones debe conocer qué ocurre y por qué, y a su vez conocer su propia dinámica y el contexto en el que se desenvuelve (Entrevistado 10); [...] La información externa ofrece el panorama del contexto externo en que está insertada la organización, permite percibir los factores políticos, económicos y sociales que pueden influir en la vida diaria de la organización. La información interna, por su parte, permite conocer la vida organizacional, el qué se está haciendo, el cómo, el cuándo, y sobre todo, los resultados (Entrevistado 11); [...] La validación de las fuentes es fundamental pues permite validar la pertinencia de la información (Entrevistado 3).

\section{Información crítica o relevante}

Si bien el rol de la información es determinante para la toma de decisiones y como bien plantean todos los directivos (100\%), dependiendo de la situaciónproblema que se presente, se identifica y determina 
la información necesaria, lo cierto es que los directivos tienen preferencias ante informaciones puntuales que se consultan y utilizan por su impacto en los procesos de decisión (Figure 2).

Entre los tipos de información que se destacan como muy importante se encuentra la información estratégica, la jurídica y la económica, y en menor medida la información sobre recursos humanos, la científico técnica y la información sobre el contexto externo. A continuación se presentan algunos de los criterios que sustentan estos resultados:

La información estratégica es importante pues se debe conocer cuáles son las prioridades de la organización, sus objetivos, misión, visión, estrategia a corto, mediano y largo plazo ya que en base a ésta se toman decisiones (Entrevistado 2); [...] La información jurídica permite identificar lo que está regulado o normado en la organización y su ambiente externo, de forma que la decisión que se tome esté en correspondencia con esto (Entrevistado 7); [...] Hoy en día, la información económicaes relevante parasaberquéalternativas debes tomar e implementar (Entrevistado 4); [...] Los directivos deben conocer la información económica asociada a su organización, al menos tener un buen panorama sobre la misma (Entrevistado 2).

\section{Problemáticas asociadas al uso de información}

Uno de los elementos importantes a tener en cuenta en los procesos de decisión son sus problemáticas de uso. Indiscutiblemente, cuando se maneja información se generan problemas que pueden visualizarse con mayor o menor dificultad. Entre los más frecuentes se identifican: la cantidad y reconocimiento de la información a la hora de tomar decisiones, la seguridad y confiabilidad de la misma, así como el acceso que en ocasiones limita los procesos de búsqueda y recuperación/selección. Algunos de los criterios que sustentan estos resultados son:

Para procesar información hay que tener determinadas habilidades y competencias que no siempre se tienen (Entrevistado 1); [...] A veces el exceso de información no es favorable pues retarda el proceso de decisión y suele ocurrir que es difícil desechar qué información no es útil. Por otro lado contar con menos información puede implicar que no se tengan en cuenta informaciones que pueden ser importantes para tomar decisiones (Entrevistado 9); [...] En ocasiones no se tiene conocimiento de la importancia de la información interna y externa en el proceso de decisiones (Entrevistado 9); [...] Algunas veces cuesta mucho trabajo acceder a determinadas fuentes de información (Entrevistado 10).

\section{Factores organizacionales que intervienen en la toma de decisiones estratégicas}

Las entrevistas realizadas permitieron identificar factores organizacionales que podían incidir positiva o negativamente, dependiendo del uso que se hiciera de éstos y la proyección organizacional ante los mismos. Esto se percibe cuando se determinan entre los factores: estrategia organizacional, relación organización-contexto, infraestructura tecnológica, cultura organizacional, cultura informacional, capacitación y capital humano. Lo positivo o negativo de cada uno de estos factores radica en el uso y comportamiento que se tenga a nivel organizacional, es por eso que los criterios se sustentan, como resultados alcanzados, en la forma y pueden o no favorecer los procesos de decisión

Entre los criterios que sustentan los factores positivos se encuentran:

Una visión estratégica consensuada y alienada entre organizaciones de información y sus 
organismos rectores puede ser favorable (Entrevistado 2); [...] Una buena cultura organizacional genera sentido de pertenencia, motivación y buen clima laboral, así como la existencia de líderes de conocimiento (Entrevistado 6); [...] La cultura informacional permite contar con competencias informacionales y buenos hábitos y costumbres en cuanto al uso de información (Entrevistado 5); [...] Un buen trabajo con el capital humano facilita implicarlos en los procesos de decisión facilitando estabilidad y confianza para tomar decisiones (Entrevistado 11); [...] Una buena infraestructura tecnológica contribuiría a gestionar toda la información interna de la organización (Entrevistado 3).

Entre los criterios que sustentan los factores negativos se encuentran:

Sin una adecuada estrategia organizacional no se pueden establecer acciones y decisiones de forma proactiva (Entrevistado 9); [...] una inadecuada relación de la organización con su contexto externo no permite crear una estrategia conjunta para el cumplimiento y alcance de una visión y objetivos estratégicos del sector (Entrevistado 2); [...] Una mala infraestructura tecnológica genera mal uso de la información, de los recursos y procesos informativos (Entrevistado 5); [...] La falta de una buena cultura organizacional e informacional impide que exista sentido de pertenencia, y trae aparejada inadecuados hábitos, así como la existencia de líderes negativos (Entrevistado 8).

\section{Procesos informacionales}

Entre los procesos informacionales que se identifican en el estudio, y que en la opinión de los entrevistados intervienen en la toma de decisiones estratégicas se encuentran la Búsqueda y Selección de Información (100\%) y el Análisis e Interpretación de la misma (100\%). También se identifican como procesos necesarios la Identificación de necesidades informativas (63\%) y la organización/procesamiento de información (63\%). Estos resultados corroboran que en los procesos de decisión se destacan aquellos procesos relacionados con la identificación, búsqueda y selección, organización y análisis para generar alternativas de solución y planes de acción de forma efectiva (Figure 3).

Algunos de los criterios emitidos que respaldan los procesos identificados se presentan a continuación:

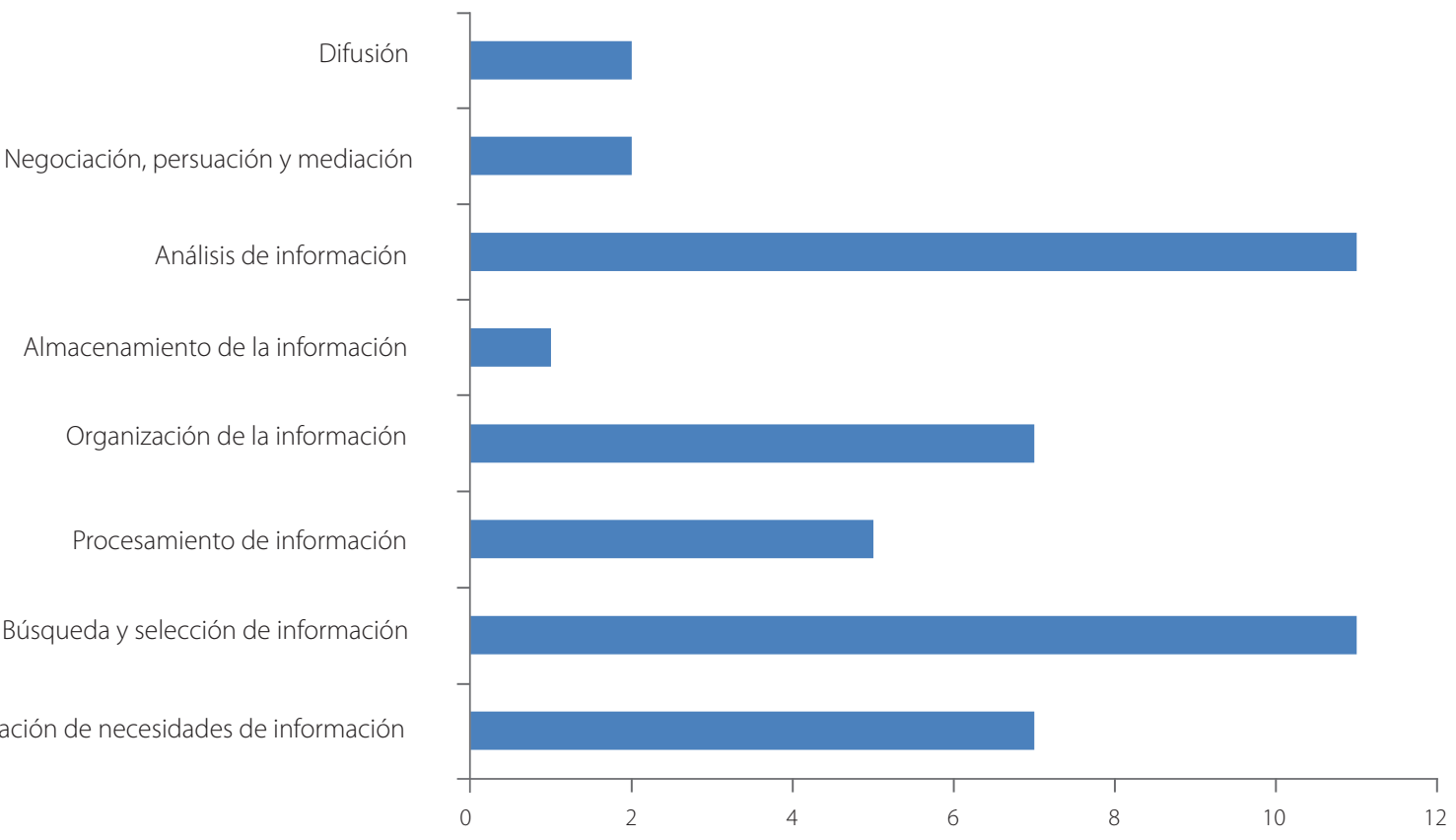

Figure 3. Cantidad de directivos que identifican los procesos informacionales que intervienen en la toma de decisiones estratégicas. 
Antes de buscar hay que definir qué y para qué, es decir, las necesidades que se tienen (Entrevistado 2); [...] La búsqueda y selección de información es uno de los procesos clave pues garantiza identificar, localizar, buscar y disponer de la información necesaria en el processo (Entrevistado 5); [...] La organización de información y su análisis permiten asignarle valor a los datos que se tienen, y por tanto se puede percibir bien o mal la situación y el contexto (Entrevistado 10); [...] El análisis es fundamental ya que no se puede interpretar la información e identificar soluciones sin crear conocimiento, sin que se intercambie $y$ comparta información (Entrevistado 11).

\section{Procesos gerenciales}

En la literatura especializada sobre la toma de decisiones organizacionales, se plantea que la misma está mediada o condicionada por determinados procesos gerenciales de apoyo. Entre los procesos gerenciales de apoyo que se identifican en la presente investigación se encuentran la Gestión Organizacional (73\%), Gestión Documental (100\%), de Información (100\%) y del Conocimiento (90\%), así como la Inteligencia Organizacional (55\%).

La Gestión Organizacional contribuye a alinear el proceso de decisión con los objetivos estratégicos y capacidades de la organización (Entrevistado 3); [...] La Gestión Documental permite que los documentos archivísticos se utilicen de forma que se pueda contar con información auténtica y veraz, y garantiza que se pueda documentar el proceso de decisión para su uso en escenarios futuros (Entrevistado 7); [...] La Gestión de Información permite que todos los sistemas de información de la organización funcionen de manera coordenada (Entrevistado 9); [...] ayuda a tener claridad de los flujos informacionales y los recursos que intervienen en la toma de decisiones (Entrevistado 2); [...] La Gestión del Conocimiento garantiza que se desarrollen de forma adecuada los procesos de conocimiento que tienen lugar en la toma de decisiones (Entrevistado 5); [...] La Vigilancia, la Prospectiva y la Inteligencia Competitiva son necesarias pues permiten explorar el ambiente externo para poder disponer de información externa de relevancia para las organizaciones de información (Entrevistado 9).

\section{Estados emocionales}

Entre los directivos entrevistados, el 100\% considera que tomar decisiones implica estar bajo determinados estados emocionales positivos y negativos, dependiendo de la situación-problema y su complejidad.

Entre los estados emocionales positivos se identifican la satisfacción, certidumbre, alegría y tranquilidad. Los mismos se experimentan cuando los individuos perciben que se tomó una buena decisión y que se hizo buen uso de la información disponible al apoyarse en un sistema de información que facilite la consulta a la misma. A continuación se presentan algunos de los criterios que sustentan estos estados emocionales:

Se experimenta una satisfacción plena y gran bienestar profesional y personal cuando se toma una decisión adecuada (Entrevistado 6); [...] Hay certidumbre cuando se cuenta con información, se tienen claros los indicadores para evaluar la actividad y existe un buen Sistema de Información (Entrevistado 3); [...] el directivo experimenta tranquilidad si cuenta con información y existe un buen Sistema de Información Integrado (Entrevistado 2).

Entre los estados emocionales negativos se identifican la incertidumbre, estrés y depresión. Los mismos se experimentan asociados a factores como el tiempo, falta de información, ausencia de un buen sistema de información y decisiones desacertadas. Al respecto se presentan algunos de los criterios que emiten los entrevistados:

\begin{abstract}
A veces se experimenta desesperación e incertidumbre asociadas a la falta de información por el factor tempo (Entrevistado 8); [...] Cuando la información es mucha pero no es precisa se genera un poco de estrés. A veces suele ser tanta que al no estar resumida y procesada se incrementa este estado (Entrevistado 5); [...] A veces se experimenta depresión cuando se tiene gran expectativa de la información y no se obtiene la que se desea (Entrevistado 3).
\end{abstract}

\section{Sistemas de información}

La utilidad y apoyo en Sistemas de Información es fundamental para el 100\% de los entrevistados. Al respecto, la totalidad de éstos coincide en que la toma 
de decisiones en instituciones de información debe apoyarse en un Sistema de Información Organizacional que permita el desarrollo de todos los procesos informacionales, así como el acceso y uso de las fuentes e informaciones que éste utiliza. Este resultado se contrasta en los siguientes criterios emitidos por los entrevistados:

Debe existir un sistema de información en el que se integre la información interna y externa de la organización. Que establezca indicadores para cumplir los objetivos y actualice la información en cuanto a éstos. Que tenga integrado un Sistema - Subsistema de Gestión Documental con toda la documentación archivística y Sistemas de Vigilancia Tecnológica e Inteligencia Empresarial que faciliten y permitan un monitoreo del entorno o ambiente externo (Entrevistado 2); [...] Que garantice la organización y flujo de toda la información asociada a los procesos y actividades del centro (Entrevistado 5).

\section{Técnicas y herramientas}

El uso de información en la toma de decisiones a nivel estratégico está condicionado por las técnicas y herramientas que desarrollan los directivos en las organizaciones de información. Entre las que se identificaron en el estudio destacan la Vigilancia Tecnológica y el monitoreo de información, el Benchmarking y la tormenta de ideas. Al respecto se presentan los criterios que sustentan esta consideración:

La Vigilancia tecnológica y el monitoreo de información permitirían obtener información relevante del ambiente externo y los competidores (Entrevistado 10); [... El Benchmarking permitiría disponer de información relevante asociada a buenas prácticas de otras organizaciones que incidiría en la toma de decisiones organizacionales (Entrevistado 8); [...] La tormenta de ideas facilitaría el intercambio de criterios y conocimiento (Entrevistado 6).

\section{Competencias informacionales}

La toma de decisiones constituye un proceso de aprendizaje continuo. En el desarrollo de la misma se pueden establecer programas de formación y capacitación que permitan la creación de conocimiento o competencias desde el plano formal, aunque empíricamente los directivos crean sus propios modelos y patrones para ejecutar el proceso. Es por ello que entre las competencias informacionales se identifican: las asociadas con el desarrollo de los procesos informacionales, los procesos gerenciales, las capacidades de análisis, el dominio de herramientas gerenciales y de las nuevas tecnologías de información y comunicación. Se visualizan competencias que no sólo pueden crearse en espacios y programas formales (capacitación), sino también de forma informal como lo constituye el aprendizaje individual.

\section{Profesional de información}

El profesional de información es valorado como útil por la totalidad de los entrevistados y ésto está dado por sus competencias informacionales. Este especialista puede desarrollar procesos gerenciales e informacionales y garantizar un adecuado flujo de información estratégica. A continuación se muestran algunos de los criterios que sustentan esta afirmación:

El profesional de información puede apoyar la toma de decisiones estratégicas garantizando los flujos de información en cada nivel de decisión (Entrevistado 1); [...] Su rol puede orientarse como gestor de información, de esta manera estaría organizando, controlando y estructurando la información para que se use adecuadamente por los directivos (Entrevistado 11).

\section{Discussão}

A partir de los resultados obtenidos en el estudio se pudo constatar que existen una serie de elementos, procesos y factores que condicionan el adecuado uso de información en la toma de decisiones estratégicas. El siguiente mapa conceptual y discursivo enfatiza los requerimientos informacionales y el modo en que éstos inciden en un adecuado proceso de decisión a nivel estratégico.

El mapa conceptual discursivo (Figure 4) que se presenta permite identificar elementos y factores que inciden en el uso de información para la toma de 


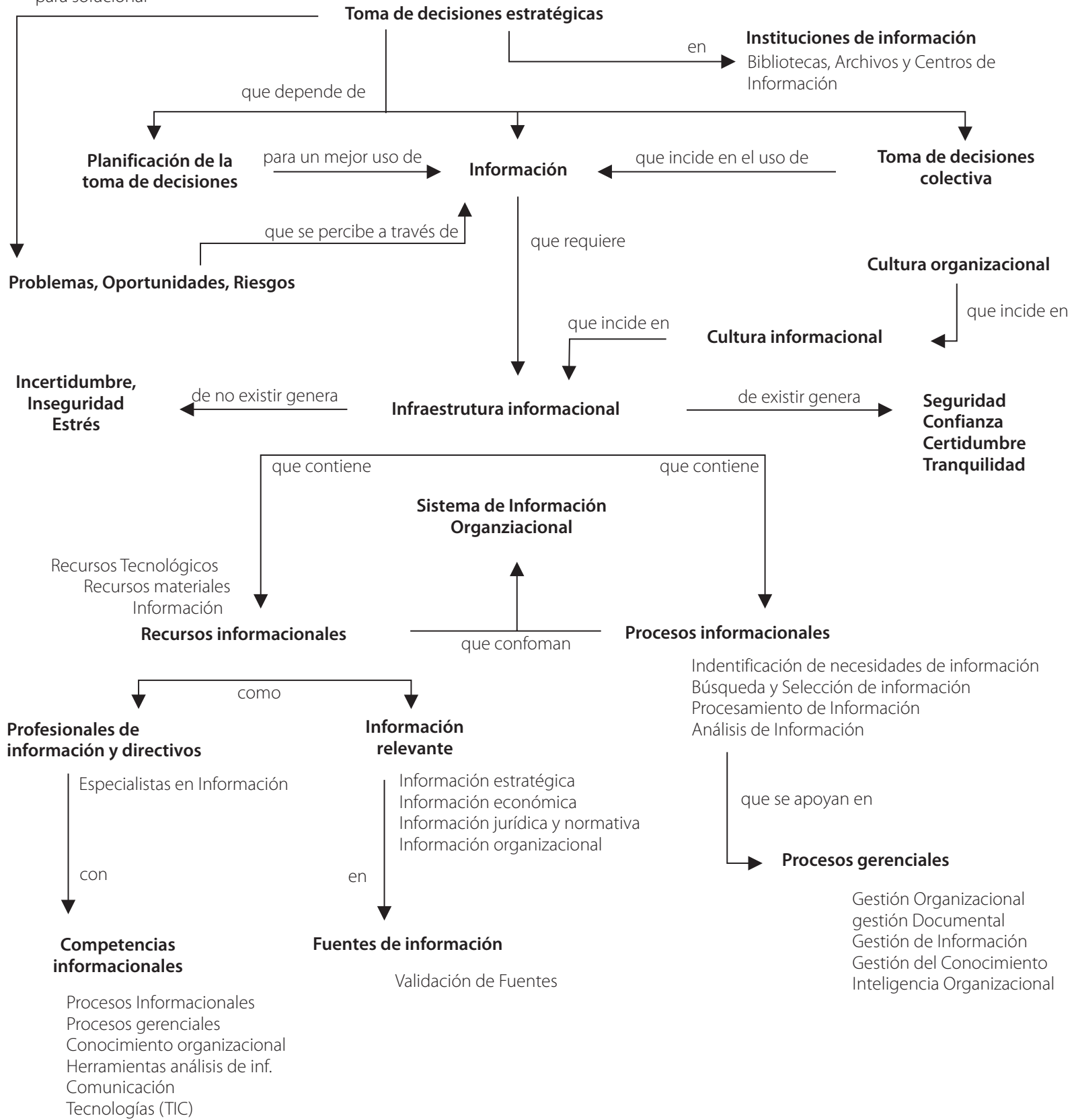

Figure 4. Mapa conceptual y discursivo sobre los requerimientos informacionales para la toma de decisiones estratégicas.

Fuente: Elaboración propia (2014).

decisiones. La información se reconoce como recurso estratégico que condiciona el carácter informacional de este proceso, sustentado por autores como Bettis (2012), Citroen (2011), Rodríguez-Cruz (2013), Rodríguez-Cruz y Pinto (2010).
En el proceso se identifican las tres situacionesproblema que mayor incidencia tienen en el mismo como bien plantean Rodríguez-Cruz y Pinto (2010).

La información relevante que se identifica coincide con los criterios y tipología presentados por 
Valentim (2006) y los estados emocionales presentados articulan con los criterios de confiabilidad, autenticidad y veracidad abordados Ponjuán et al. (2014). Estos estados emocionales respaldados por Almeida y Sobral (2005), resaltan el papel de las emociones en la negociación y sus resultados positivos.

Los procesos informacionales que se identifican, examinados por Rodríguez-Cruz y Pinto (2010), se apoyan en los sistemas de información que sirven de apoyo a la toma de decisiones. En este sentido, como bien plantean Laudon y Laudon (2012), la concepción de sistemas de información desde una perspectiva gerencial incide de forma notable en la toma de decisiones estratégicas.

Por otra parte, existen un conjunto de procesos gerenciales que requieren una adecuada articulación para obtener beneficios que ayuden en la toma de decisiones. Entre éstos se encuentran la Gestión Organizacional, Documental, de Información y del Conocimiento, así como la Inteligencia. Estos procesos particulares, y su vínculo con los procesos de decisión, han sido abordados por autores como Martins y Miranda (2011), Wright (2013), Ponjuán et al. (2014).

\section{Conclusiones}

La toma de decisiones estratégicas requiere un conjunto de componentes, elementos y factores informacionales que inciden en su adecuado desarrollo. A partir de la utilización del método fenomenográfico se pudo identificar estos aspectos y construir un mapa conceptual discursivo para la adecuada proyección del contexto y escenario informacional para tomar decisiones estratégicas.

Los requerimientos informacionales identificados y reflejados en el mapa conceptual discursivo validan los referentes teóricos examinados en el presente estudio. De esta forma se valida el carácter informacional de la toma de decisiones a partir de su dependencia de la información como recurso estratégico. El proceso se orienta indistintamente a la solución de problemas, aprovechamiento de oportunidades y prevención de riesgos a partir de una toma de decisiones grupal, en correspondencia con el denominado 'dominio de emergencias' que se presenta en el proceso. Esta toma de decisiones grupales reduce el riesgo y la incertidumbre a partir de una efectiva infraestructura informacional.

Dicha infraestructura informacional se basa en un contexto informacional con recursos, estrategias, competencias y una cultura informacional que propician una rápida adaptación a partir del uso estratégico de información, como reflejo de las denominadas 'capacidades dinámicas organizacionales' determinadas por estos mismos autores.

Entre los requerimientos informacionales se identifican las fuentes y los ambientes informacionales (externos e internos) y la necesidad de su validación, así como criterios y componentes que garantizan la efectividad en el uso de información y evitan una 'sobrecarga de información'. Entre éstos se destacan los tipos de información que intervienen en el proceso, las cualidades de la información, los recursos para su tratamiento y uso, y los procesos informacionales que garantizan no solo la calidad, sino la cantidad, variedad y procesamiento de fuentes de información.

Como parte de ese contexto informacional, tal y como se evidencia en los referentes teóricos examinados, se hace necesaria la existencia de sistemas de información que permitan desarrollar los procesos informacionales asociados a la toma de decisiones. De igual forma se identifican como procesos estratégicos de apoyo a la Gestión Organizacional, Documental, de Información, del Conocimiento e Inteligencia Organizacional, bajo los cuales se puede obtener información relevante y de obligada consulta, previa validación de las fuentes de información que se utilizan en los procesos de decisión.

Determinar los requerimientos informacionales de la toma de decisiones constituye, por tanto, una primera aproximación para generar condiciones objetivas y subjetivas que fomenten una adecuada cultura informacional y en consecuencia un buen uso de la información en el desarrollo de estos procesos a nivel estratégico. Esclarecer y comprender estos 
requerimientos contribuye para que las instituciones de información puedan orientar mejor sus acciones y esfuerzos a fin de generar capacidades para tomar decisiones estratégicas de forma efectiva.

\section{Colaboraciones}

Todos los autores contribuyen a la concepción y diseño del estudio, análisis de datos y redacción final.

\section{Referencias}

AKGUN, A. E.; BYRNE, J.; KESKIN, H. Organizational intelligence: A structuration view. Journal of Organizational Change Management, v. 20, n. 3, p. 272-289, 2007.

ALLEN, D. Information Behavior and decision making in time constrained practice: A dual-processing perspective. Journal of the American Society for Information Science and Technology, v. 62, n. 11, p. 2165-2181, 2011.

ALMEIDA, F. J. R.; SOBRAL, F. J. B. A. Emoções, inteligência e negociação: um estudo empírico sobre a percepção dos gerentes portugueses. RAC: Revista de Administração Contemporânea, v. 9, n. 4, p. 9-30, 2005.

ANGELONI, M. T. Elementos intervenientes na tomada de decisão. Ciência da Informação, v. 32, n. 1, p. 17-22, 2003.

ARIÑO, M. A. Toma de decisiones y gobierno de organizaciones. Barcelona: Ediciones Deusto, 2005.

BETTIS, H. Decision-making's impact on organizational learning and information overload. Journal of Business Research, v. 65, p. 814-820, 2012.

CAIXETA, M. L.; RODRIGUES, R. B. A decisão como resultado de um processo social da informacão compartilhada. Informação \& Informação, v. 13, n. 1, p. 81-104, 2008.

$\mathrm{CHOU}, \mathrm{H}$.; LIN, Y.; CHOU, S. Team cognition, collective efficacy, and performance in strategic decisión making teams. Social Behavior and Personality, v. 49, n. 3, p. 381-394, 2012.

CITROEN, C. The role of information in strategic decisionmaking. International Journal of Information Management, v. 31, p. 493-501, 2011.

DIEHM, R. A.; LUPTON, M. Approaches to Learning Information Literacy: A Phenomenographic study. The Journal of Academic Librarianship, v. 38, n. 4, p. 217-225, 2012.

FIOL, M. La toma de decisiones de directivos latinos. RAE: Revista de Administração de Empresas, v. 41, n. 4, p. 16-25, 2001.

HERNÁNDEZ, R.; FERNÁNDEZ, C.; LUCIO, M. P. B. Metodología de la Investigación. 5. ed. Colombia: McGraw Hill, 2010.

HOWARD, A.; ORTIZ, S. Decision Making and the Study of Social Process. Acta Sociológica, v. 14, p. 213-226, 1971.

HUNG, Y.; HUANG, H. J.; GOSLING, M. Deviation and escalation: Decision-making pitfalls illustrated. Journal of Organizational Change Management, v. 24, n. 5, p. 695-711, 2011.
JANSEN, J. G. et al. Social capital as a decision aid in strategic decision-making in service organizations. Management Decision, v. 49, n. 5, p. 695-711, 2011.

LAUDON, K.; LAUDON, J. Sistemas de Información Gerencial. 12. ed. Colombia: Pearson Education, 2012.

MARTINS, J. B.; MIRANDA, R. C. R. Aprimoramento de estrategistas e decisores novatos: a gestão do conhecimento estratégico aplicada ao Centro de Apoio ao Desenvolvimento Tecnológico da Universidade de Brasília. TransInformação, v. 23, n. 2, p. 139-157, 2011. http://dx.doi.org/10.1590/S0103-3 7862011000200005

McKENZIE, J.; VAN WINKELEN, C.; GREWAL, S. Developing organisational decision-making capability: A knowledge manager's guide. Journal of Knowledge Management, v. 15, n. 3, p. 403-421, 2011.

MINTZBERG, H.; RAISINGHANI, D.; THĖORÊT, A. The structure of "unstructured" decision processes. Administrative Science Quarterly, v. 21, n. 2, p. 247-275, 1976.

MITCHELL, J. R.; SHEPHERD, D. A.; SHARFMAN, M. P. Erratic strategic decisions: When and why managers are inconsistent in strategic decisión making. Strategic Management Journal, v. 32, p. 683-704, 2011.

PONJUÁN, G.; MENA, M.; RODRÍGUEZ-CRUZ, Y. Fundamentos de la Gestión Documental, de Información y del Conocimiento. La Habana: Editorial Félix Varela, 2014.

RODRÍGUEZ, E.; PEDRAJA, L. Análisis del impacto del proceso de toma de decisiones estratégicas sobre la eficacia de las organizaciones públicas. Innovar, v. 19, n. 35, p. 33-46, 2009.

RODRÍGUEZ-CRUZ, Y. El impacto de la racionalidad limitada en el proceso informacional de toma de decisiones organizacionales. Revista Cubana de Información en Ciencias de la Salud, v. 24, n. 1, p. 56-72, 2013.

RODRÍGUEZ-CRUZ, Y.; PINTO, M. Evolución, particularidades y carácter informacional de la toma de decisiones organizacionales. ACIMED, v. 21, n. 1, p. 57-77, 2010

SILVA, E. R. et al. Processamento cognitivo da informacão para tomada de decisão. Perspectivas em Gestão: Conhecimento, v. 1, n. 1, p. 25-39, 2011.

SIMON, H. A. A behavioral Model of Rational Choice. The Quarterly Journal of Economics, v. 69, n. 1, p. 99-118, 1955. 
VALENTIM, M. L. P. Informacão, conhecimento e inteligência organizacional. Marília: Fundepe, 2006.

WILSON, D. C. et al. Extreme events, organizations and the politics of strategic decision making. Accounting, Auditing \& Accountability Journal, v. 23, n. 5, p. 699-721, 2010.
WRIGHT, T. Information culture in a government organization: Examining records management training and self-perceived competencies in compliance with a records management program. Records Management Journal. v. 23, n. 1, p. 14-36, 2013.
189

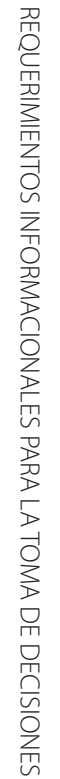


